Editora Omnis Scientia

ANAIS DA I JORNADA NORTE-NORDESTE DE ENGENHARIAS (ONLINE)

Volume 1

$1^{\text {a }}$ Edição

TRIUNFO - PE 


\section{Coordenador Científico}

Alex Aguiar da Silva

\section{Coordenador de Publicação}

Daniel Luís Viana Cruz

\section{Coordenadora do Evento}

Andréa Telino Gomes

\section{Organizadores}

Academics - Eventos acadêmicos online

Editora Omnis Scientia

Andréa Telino Gomes

Daniel Luís Viana Cruz

Alex Aguiar da Silva

\section{Palestrantes}

Alex Aguiar da Silva

André Búrigo Leite

Cecília Aguiar da Silva

Eugênio Bastos da Costa

Jorge Recarte Henriquez Guerrero

Renan Ferreira da Rocha

\section{Avaliadores}

Alex Aguiar da Silva

Helio Cardoso Martim

Péricles Felipe Bastos Gomes

\section{Imagem de Capa}

Freepik

\section{Revisão}

Os autores

\section{(9) $\mathbb{\oplus \Theta \Theta}$}

Este trabalho está licenciado com uma Licença Creative Commons - Atribuição-NãoComercialSemDerivações 4.0 Internacional.

O conteúdo abordado nos artigos, seus dados em sua forma, correção e confiabilidade são de responsabilidade exclusiva dos autores. 


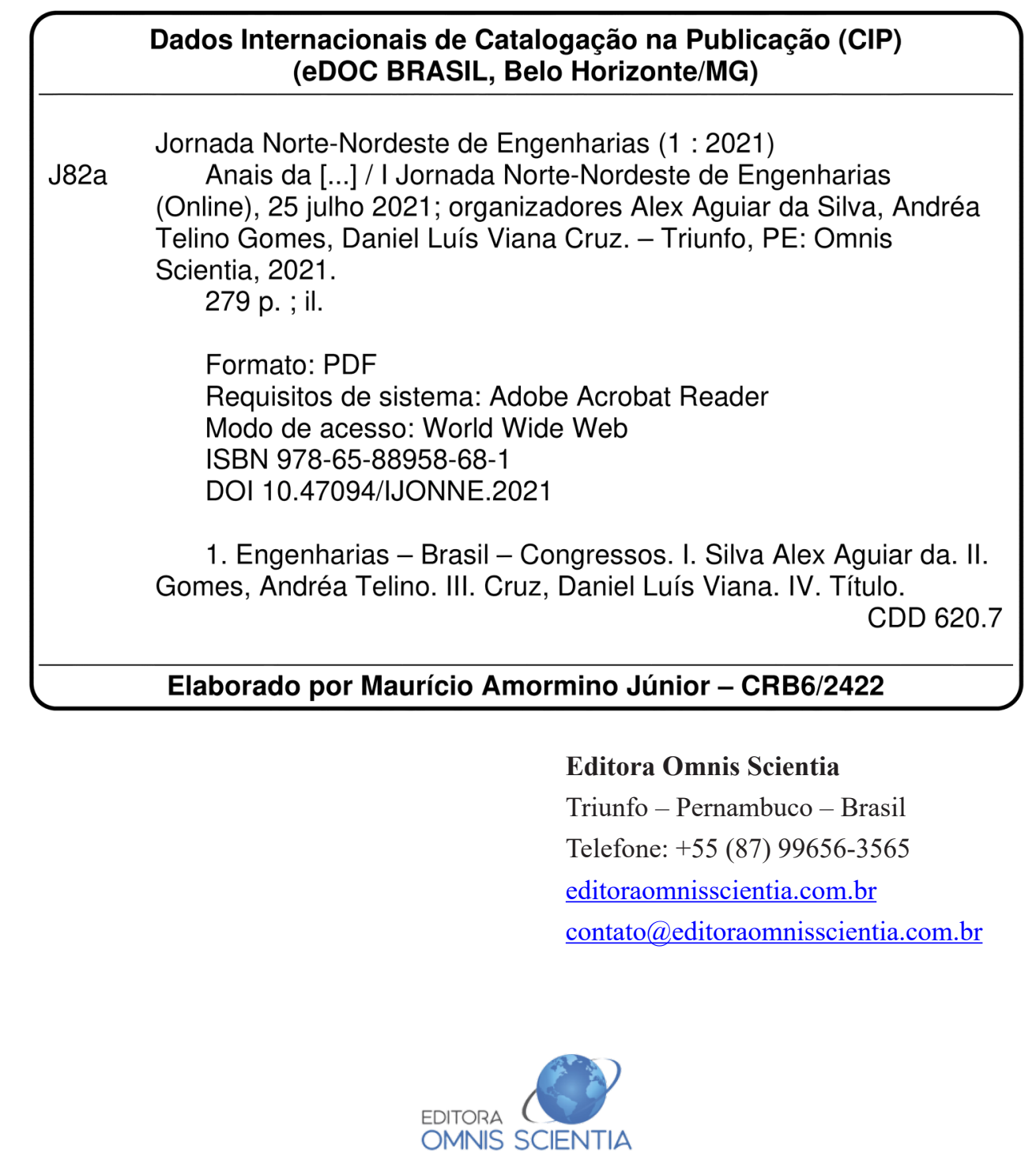




\section{EDITORIAL}

A grande área das engenharias abrangente uma série de ramos específicos, cada qual com determinados campos de aplicação e tipos de tecnologias, conjugando conhecimentos especializados no sentido de viabilizar as utilidades, tendo em conta a sociedade, a técnica, a economia e o meio ambiente. Deste modo, as engenharias aplicam o conhecimento científico, econômico, social e prático, para criar, desenhar, construir, manter e melhorar estruturas, máquinas, aparelhos, sistemas, materiais e processos. Assim, se adquire e se aplicam os conhecimentos matemáticos e técnicos na invenção, aperfeiçoamento e implementação de utilidades que realizem uma função ou objetivo.

A I Jornada Norte-Nordeste de Engenharias (online) I JONNE, objetivou reunir e integrar as engenharias para promover e apoiar esta área do conhecimento por meio de palestras, submissões de trabalhos na modalidade de resumos expandidos e exposição dos resumos aprovados.

Os participantes receberam certificados de participação de 20 horas e foi concedido menção honrosa para os três melhores trabalhos. Seguem os títulos dos resumos que foram premiados:

Modalidade resumo expandido

387210 - DIMENSIONAMENTO DE TROCADOR DE CALOR A PLACAS PARA PASTEURIZAÇÃO DE LEITE PELO PROCESSO HTST

387222 - DESENVOLVIMENTO DE UM DISPENSER DE ÁLCOOL GEL AUTOMÁTICO PARA MONITORAMENTO DA COMPLACÊNCIA DE MÃOS

387225 - REDE NEURAL ARTIFICIAL PARA VISCOSIDADE E CONDUTIVIDADE TÉRMICA DO FLUIDO REFRIGERANTE R32

A equipe organizadora da I JONNE agradece a todos os participantes, palestrantes e avaliadores pela participação e colaboração no congresso. 


\section{SUMÁRIO}

\section{ENGENHARIA CIVIL}

ANÁLISE DO USO DE RESÍDUO DE BRITAGEM DAS ROCHAS EM CONCRETO AUTOADENSÁVEL: UMA REVISÃO DA LITERATURA.....................................................12

ESTRATÉGIAS PARA A PREVENÇÃO DE ACIDENTES NA CONSTRUÇÃO CIVIL: UMA REVISÃO SISTEMÁTICA DA LITERATURA.

RESISTÊNCIA À COMPRESSÃO DE MISTURAS DE SOLO-CIMENTO COM NANOSSÍLICA E SÍLICA ATIVA.

PATOLOGIAS EM OBRAS PARALISADAS: O CASO DO ESTÁDIO COLOSSO DO TAPAJÓS NA CIDADE DE SANTARÉM - PA.

DIFERENÇA NO CUSTO DE MÃO DE OBRA EM FABRICAÇÃO DE LAJES PRÉ- MOLDADAS EM AMBITO NACIONAL

ESTUDO COMPARATIVO DE FUNDAÇÕES PARA UM EDIFÍCIO MODELO EM JOÃO PESSOA/PB: SAPATA X ESTACA HÉLICE CONTÍNUA.

MANIFESTAÇÕES PATOLÓGICAS EM EDIFICAÇÕES COM PAREDES DE CONCRETO ARMADO: ESTUDO DE CASO EM OBRA NA CIDADE DE JOÃO PESSOA - PB.....

REUTILIZAÇÃO DO PÓ RESIDUAL DE MARMORARIA PARA SUBSTITUIÇÃO DO AGREGADO MIÚDO NO CONCRETO.

SUBSIDÊNCIA OCORRIDA NOS BAIRROS PINHEIRO, BEBEDOURO E MUTANGE EM MACEIÓ - AL: UMA REVISÃO BIBLIOGRÁFICA.

CARACTERIZAÇÃO FÍSICA DAS VARIAÇÕES DIMENSIONAIS DO BAMBU GUADUA WEBERBAUERI DE RIO BRANCO - AC.

ESTUDO DA OCORRÊNCIA DE SOLOS COLAPSÍVEIS NA REGIÃO NORDESTE DO BRASIL.

OS EFEITOS DO USO DE POÇOS ARTESIANOS NO PROCESSO DE SALINIZAÇÃO COSTEIRA NA CIDADE DE SÃO LUÍS: UM LEVANTAMENTO BIBLIOGRAFICO.

TEORIADOS SÓLIDOS CELULARES EMPREGADOS EM REFORÇOS PARAACONSTRUÇÃO CIVIL

AVALIAÇÃO DO EXTRATO DE BOLDO E AROEIRA COMO INIBIDOR VEGETAL DE BAIXO CUSTO......

ANÁLISE DE DOSAGEM DE MATRIZ CIMENTÍCIA COM FIBRAS DE POLIPROPILENO: REVISÃO DA LITERATURA 
ANÁLISE DA RETENÇÃO DE CLORETOS EM ARGAMASSAS COM RESÍDUOS DE CELULOSE.

\section{ENGENHARIA DE MATERIAIS E METALÚRGICA}

ANÁLISE DA DEGRADAÇÃO FERRÍTICA DO AÇO INOXIDÁVEL DUPLEX SAF 2205 SUBMETIDO A ENVELHECIMENTO ISOTÉRMICO.

ANÁLISE DE INCLUSÕES E QUANTIFICAÇÃO DE POROSIDADE DO BRONZE AO ESTANHO EM ESTRUTURA BRUTA DE FUSÃO. 100

SÍNTESE RÁPIDA DE NANOFIOS DE NIOBATO DE SÓDIO. 105

AVALIAÇÃODAINCORPORAÇÃODACAREPADELAMINAÇÃOCOMOPARTEDAMATÉRIAPRIMA NA PRODUÇÃO DE SÍNTER DE FINOS DO MINÉRIO DE FERRO. 110

ESTUDO PROSPECTIVO DO PETRÓLEO APLICADO EM NANOMATERIAL CERÂMICO...... 115

ESTUDO PROSPECTIVO DE NANOMATERIAL CERÂMICO APLICADO EM SAÚDE MÉDICA E DENTÁRIA.

ESTUDO PROSPECTIVO DE MAGNETITA NANOMÉTRICA COMO MATERIAL FERROMAGNETICO.

ESTUDO PROSPECTIVO DE CERÂMICA AVANÇADA UTILIZANDO DOPAGEM......

BUSCA DE ANTERIORIDADE DE CERÂMICA APLICADA NA REMOÇÃO DE CONTAMINANTE..... .131

APLICAÇÕES DE $\mathrm{MoO}_{3}$ OBTIVO ATRAVÉS DE PRECIPITAÇÃO. 136

REVISÃO BIBLIOGRÁFICA SOBRE TUNGSTATO DE BÁRIO EM MEIO AQUOSO. 140

ESTUDOS DA DIFUSÃO DE CARBONO NOS AÇOS SAE 1020 E 1045 SUBMETIDOS À TRATAMENTO TERMOQUÍMICO DE CEMENTAÇÃO SÓLIDA. 145

\section{ENGENHARIA DO PRODUTO}

DESENVOLVIMENTO DE UM DISPENSER DE ÁLCOOL GEL AUTOMÁTICO PARA MONITORAMENTO DA COMPLACÊNCIA DE MÃOS. 150

\section{ENGENHARIA ELÉTRICA}

ENERGIA EÓLICA NO CEARÁ .156

\section{ENGENHARIA HIDRÁULICA}

ESTUDO DE MÉTODOS ANALÍTICOS PARA DETERMINAÇÃO DE PERDA DE CARGA EM TUBULAÇÕES DE PVC UTILIZADAS EM IRRIGAÇÕES. 162 
DESENVOLVIMENTO DE PRÁTICAS LABORATORIAIS APLICANDO OS CONCEITOS DE FENÔMENOS DE TRANSPORTES. 168

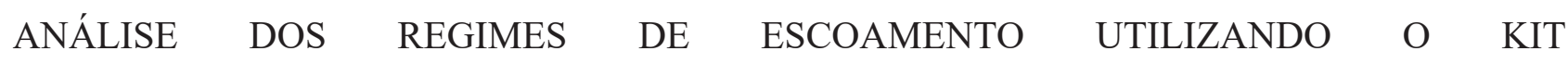
AQUALIBRIUM...

\section{ENGENHARIA MECÂNICA}

AVANÇO NO EMPREGO DE GABARITOS DE SOLDAGEM NO PROCESSO DE FABRICAÇÃO DE CHASSIS FORMULA SAE..

ANÁLISE DA DISTRIBUIÇÃO DE TEMPERATURA NOS PNEUS DE UM VEÍCULO FORMULA SAE PARA DIFERENTES CARGAS VERTICAIS. 184

\section{ENGENHARIA QUÍMICA}

UTILIZAÇÃO DE OXIDAÇÃO AVANÇADA PARA O TRATAMENTO DE EFLUENTES INDUSTRIAIS: UMA REVISÃO DA LITERATURA..

ANÁLISE DOS PRINCIPAIS PROCESSOS DE PRODUÇÃO DE BIODIESEL: UMA REVISÃO DE LITERATURA.

A QUÍMICA DOS AGROTÓXICOS USADOS EM AGRICULTURA: UMA REVISÃO DA LITERATURA. 201

AVALIAÇÃO CINÉTICA DA REAÇÃO DE DEGRADAÇÃO DE DIURON PRESENTE EM ÁGUAS SUPERFICIAIS.

ESTUDO DE CASO DO TRATAMENTO DE GÁS ÁCIDO COM COLUNA DE ABSORÇÃO DE LEITO RECHEADO.

ELABORAÇÃO DA CURVA DE CALIBRAÇÃO PARA ACRILAMIDA PELO MÉTODO DE CROMATOGRAFIA LÍQUIDA DE ALTA EFICIÊNCIA.

DETERMINAÇÃODECONSTANTESCINÉTICASPELOS MÉTODOSINTEGRALEDERUNGEKUTTA PARA REAÇÃO DE OXIDAÇÃO DO ANTRACENO.

DIMENSIONAMENTO DE TROCADOR DE CALOR A PLACAS PARA PASTEURIZAÇÃO DE LEITE PELO PROCESSO HTST.. 229

REDE NEURAL ARTIFICIAL PARA VISCOSIDADE E CONDUTIVIDADE TÉRMICA DO FLUIDO REFRIGERANTE R32.

TRANSFORMAÇÃO DO POLITEREFTALATO DE ETILENO RECICLADO EM FIBRAS TÊXTEIS DE POLIÉSTER. .239

\section{ENGENHARIA SANITÁRIA}

O USO DA ENERGIA SOLAR EM HOSPITAIS EM TEMPOS DE PANDEMIA 
ESTUDO DE VARIÁVEIS QUE POSSAM INFLUENCIAR NO CONSUMO DE ÁGUA DA CIDADE DE BAIXO GUANDÚ-ES........

\section{OUTRAS}

SISTEMAAUTOMÁTICO DE IRRIGAÇÃO. .260

A FRUTA DO MILAGRE - SYNSEPALUM DULCIFICUM.. 266 FOSSAS DE EVAPOTRANSPIRAÇÃO: UMA SOLUÇÃO SUSTENTÁVEL PARA OS DEFICITS NO SANEAMENTO EM ÁREAS CARENTES. .271

PROJETO DE ROBÓTICA SOCIAL E EDUCACIONAL .276 


\title{
SUBSIDÊNCIA OCORRIDA NOS BAIRROS PINHEIRO, BEBEDOURO E MUTANGE EM MACEIÓ - AL: UMA REVISÃO BIBLIOGRÁFICA
}

\author{
Martha Maria Bezerra Santos¹; Iálysson da Silva Medeiros² \\ 21 \\ Mestranda em Geotecnia, Universidade Federal de Pernambuco (UFPE), Recife, \\ Pernambuco. \\ Mestrando em Geotecnia, Universidade Federal de Pernambuco (UFPE), Caruaru, \\ Pernambuco.
}

DOI: 10.47094/IJONNE.2021.2

\begin{abstract}
RESUMO
A subsidência é um fenômeno que ocorre ao redor do mundo devido a diversos fatores, como a exploração de água, gás e petróleo. Desde 2018, chamou a atenção da mídia a ocorrência acentuada desse fenômeno nos bairros de Pinheiro, Bebedouro e Mutange em Maceió - AL. Dessa forma, o objetivo desse trabalho é reunir as principais informações disponíveis na literatura sobre esse caso, por meio de uma busca na base de dados Google e Google Acadêmico. De acordo com o Serviço Geológico do Brasil - CPRM (2019) a causa da subsidência foi a exploração do sal-gema na região, e de acordo com o CNJ (2021) a solução consensual entre moradores, mineradora e o governo federal foi benéfica para todos. Com este trabalho notou-se a grande quantidade de estudos e ensaios realizados pelos profissionais envolvidos a fim de chegar a um parecer da causa do fenômeno, demonstrando responsabilidade com a investigação.
\end{abstract}

PALAVRAS-CHAVE: Afundamento; Rebaixamento do solo; Extração de Sal-gema. ÁREA TEMÁTICA: Engenharia Civil

\section{INTRODUÇÃO}

Segundo Santos (2005), subsidência é o fenômeno de rebaixamento da superfície do terreno devido a alterações sofridas pelas camadas subterrâneas, ou seja, há uma redução do nível do terreno por causa da remoção do seu suporte. Ainda segundo o autor, esse fenômeno vem ocorrendo no mundo devido aos mais diversos fatores, como a extração de água, gás e petróleo por exemplo.

Desde o ano de 2018, o caso dos bairros Pinheiro, Bebedouro e Mutange recebem a atenção da mídia devido aos danos que o afundamento das moradias e vias públicas vem causando à população, que, segundo o Serviço Geológico do Brasil - CPRM (2019), é de aproximadamente 
31.797 pessoas. Durante a investigação do processo, foram levantadas algumas hipóteses das causas desse fenômeno.

Com a abrangência da mídia acerca do assunto, o objetivo desse trabalho é realizar uma revisão bibliográfica com as informações já encontradas acerca da subsidência que ainda ocorre nos bairros Pinheiro, Bebedouro e Mutange de Maceió - AL, de forma a sintetizar as informações já disponíveis na literatura.

\section{METODOLOGIA}

Foi realizado uma pesquisa bibliográfica do tipo descritiva e de abordagem qualitativa com um estudo amplo do objeto, levando em conta a contextualização em que está inserido e as características. A amostra desse trabalho foi baseada na análise da literatura de publicação nacional sobre o caso da subsidência ocorrida nos bairros de Maceió - AL. Foram selecionadas reportagens oficiais do governo federal e estadual, artigos e relatórios entre os anos de 2019 e 2021. Para o levantamento da amostra utilizou-se a base de dados Google Acadêmico e Google por meio de uso das palavras-chave: Subsidência e Maceió.

\section{FUNDAMENTAÇÃO TEÓRICA}

Com base no estudo geológico divulgado pelo Serviço Geológico do Brasil - CPRM em 29 de abril de 2019, o bairro Pinheiro apresentava, historicamente, diversas rachaduras e afundamentos em moradias e vias públicas. Nesse mesmo estudo, é relatado que o fenômeno foi intensificado em 2018 devido as fortes chuvas de verão, no dia 15 de fevereiro de 2018 e do abalo sísmico de 2,4 mR em 3 de março de 2018.

Durante a investigação foram levantadas 4 hipóteses para as causas do problema e, com estudos utilizando o levantamento geológico-geotécnico, levantamento interferométrico, métodos geofísicos, sismologia, hidrogeologia, análise dos sonares, integração de dados geológicos e de extração de sal em ambiente 3D, chegou-se as conclusões contidas no Quadro 1. 
Quadro 1: Hipóteses, premissas e conclusões obtidas pelo CPRM através dos estudos realizados

\begin{tabular}{|c|c|c|}
\hline HIPÓTESES & PREMISSAS & CONCLUSÕES \\
\hline $\begin{array}{l}\text { 1) Características } \\
\text { geotécnicas do solo } \\
\text { da região e a forma } \\
\text { de ocupação do } \\
\text { bairro. }\end{array}$ & $\begin{array}{l}\text { Presença de solos } \\
\text { colapsíveis e } \\
\text { orgânicos, forma de } \\
\text { ocupação e métodos } \\
\text { construtivos } \\
\text { inadequados. }\end{array}$ & $\begin{array}{l}\text { Ensaios não demonstraram características } \\
\text { que explicassem os danos. Não é possível } \\
\text { justificar por problemas construtivos o } \\
\text { surgimento de rachaduras em imóveis de } \\
\text { várias idades. Essa hipótese foi invalidada } \\
\text { pelos estudos e destacou-se que a chuva } \\
\text { intensificou o processo erosivo que, } \\
\text { em processos interligados, necessita de } \\
\text { cuidados específicos. }\end{array}$ \\
\hline $\begin{array}{l}\text { 2) Presença de } \\
\text { vazios (cavidades, } \\
\text { cavernas) nos solos } \\
\text { e subsolos da região } \\
\text { decorrentes de } \\
\text { causas naturais ou } \\
\text { ações antrópicas. }\end{array}$ & $\begin{array}{c}\text { Ocorrência } \\
\text { de cavidades } \\
\text { decorrentes da } \\
\text { dissolução de rochas } \\
\text { em subsuperfícies } \\
\text { ou desabamento de } \\
\text { minas de extração } \\
\text { de sal-gema. }\end{array}$ & $\begin{array}{l}\text { Os estudos realizados mostraram uma } \\
\text { coincidência entre as minas de extração } \\
\text { e os sismos e anomalias nas cavernas } \\
\text { subterrâneas. Os sonares no ambiente } \\
\text { 3D, indicaram que a extração do sal- } \\
\text { gema alterou o estado de tensões do } \\
\text { solo, colapsando minas. Concluiu-se que } \\
\text { há evidências de que a deformação das } \\
\text { cavernas de mineração foi predominante } \\
\text { na origem dos fenômenos. }\end{array}$ \\
\hline $\begin{array}{l}\text { 3) Estruturas/ } \\
\text { Feições tectônicas } \\
\text { ativas na região. }\end{array}$ & $\begin{array}{l}\text { Os danos estudados } \\
\text { teriam origem } \\
\text { em eventos de } \\
\text { neotectônica. }\end{array}$ & $\begin{array}{c}\text { Foram identificadas falhas esperadas } \\
\text { pelo contexto regional e sismos não } \\
\text { compatíveis com movimentos tectônicos } \\
\text { regionais. Dessa forma, concluiu-se que } \\
\text { a hipótese } 2 \text { e } 3 \text { estão associadas, com } \\
\text { a hipótese } 2 \text { sendo a desencadeadora do } \\
\text { processo. }\end{array}$ \\
\hline $\begin{array}{l}\text { 4) Explotação de } \\
\text { água subterrânea. }\end{array}$ & $\begin{array}{l}\text { Os danos estudados } \\
\text { teriam origem em } \\
\text { subsidência causada } \\
\text { por recalque } \\
\text { decorrente da } \\
\text { extração de água } \\
\text { subterrânea. }\end{array}$ & $\begin{array}{l}\text { A análise dos dados indicou que os níveis } \\
\text { estáticos e dinâmicos dos aquíferos estão } \\
\text { em recuperação, de forma que não há } \\
\text { indícios de superexplotação. Ou seja, o } \\
\text { estudo realizado invalidou a hipótese }\end{array}$ \\
\hline
\end{tabular}

Fonte: Serviço Geológico do Brasil - CPRM (2019)

A mineradora realizou questionamentos sobre os estudos e algumas considerações importantes foram feitas. Quando questionados como se concluiu a relação entre instabilidade da caverna subterrânea e ativação da falha geológica, o Ministério de Minas e Energia, Secretaria de Geologia, Mineração e Transformação Mineral e Serviço Geológico do Brasil - CPRM (2019) afirmaram que não é possível estimar a energia necessária de ativação da falha geológica, ou então seria possível prever os terremotos. Entretanto, já há um consenso na literatura que a atividade humana pode estar associada a atividade sísmica, mesmo as mudanças ocorrendo em pequenas 
proporções. Segundo Kinscher et al. (2016) apud Ministérios de Minas e Energia, Secretaria de Geologia, Mineração e Transformação Mineral e Serviço Geológico do Brasil - CPRM (2019), a sismicidade devido à mineração por dissolução é uma associação feita em várias partes do mundo.

De acordo com o Conselho Nacional de Justiça (2021) o assunto sobre a subsidência dos bairros de Maceió foi citado pela primeira vez no Conselho Nacional de Justiça (CNJ) apenas em uma reunião extraordinária no dia 24 de junho de 2019. A partir disso, houve uma mobilização para assegurar o direito dos moradores e cumprir com a urgência desses mesmos moradores saírem da área afetada. Buscou-se desburocratizar e dar celeridade e sociabilidade nas ações que possibilitariam o recebimento de possíveis indenizações.

Segundo a Secretaria Adjunta Especial de Defesa Civil (2020) o monitoramento da área afetada estava sendo feito por meio de sensores Global Positioning System (GPS), interferometria, visitas e estudos de campo e vistorias, com os principais profissionais envolvidos das áreas de geologia, geografia, engenharia civil, meteorologia, engenharia de agrimensura. É ressaltado que o preenchimento das cavidades no solo é uma medida mitigadora e que, pela peculiaridade do caso, o monitoramento é a medida mais eficaz atender a população.

Vassileva et al. (2021) integrou observações de deslocamento do solo da região afetada com recursos de modelagem numérica e chegou à conclusão de que a subsidência em Maceió começou gradualmente quase duas décadas atrás, com uma aceleração lenta no início e mais rápida nos últimos 4 anos. Afirma também que o fenômeno é atribuído a uma fonte de profundidade entre 600 e 1000 m, coincidindo com as localizações das cavidades de sal e que as condições de mineração de sal ativa/pressurizada e inativa/despressurizada causaram a instabilidade mecânica das cavidades com migração local para cima.

\section{CONSIDERAÇÕES FINAIS}

Durante a elaboração do presente trabalho notou-se a grande quantidade de dados reunidos pelo CPRM (2019) para chegarem à conclusão de que a desestabilização das cavidades da extração de sal-gema foi a causa-gatilho do fenômeno de subsidência nos bairros de Maceió.

Percebeu-se também que a solução consensual adotada pela mineradora, governo federal e moradores foi a mais benéfica possível para todos os envolvidos em virtude da urgência da situação.

\section{PRINCIPAIS REFERÊNCIAS}

SANTOS, Sylvana Melo dos. Investigações metodológicas sobre o monitoramento da subsidência do solo devido à extração de água subterrânea - caso da Região Metropolitana 
de Recife. 2005. Tese (Doutorado em Ciências em Engenharia Civil) - Universidade Federal de Pernambuco, Recife, 2005.

SERVIÇO GEOLÓGICO DO BRASIL- CPRM. Estudos sobre a instabilidade do terreno nos bairros Pinheiro, Mutange e Bebedouro, Maceió (AL). Brasília: CPRM, 2019.

MINISTÉRIO DE MINAS E ENERGIA; SECRETARIA DE GEOLOGIA, MINERAÇÃO E TRANSFORMAÇÃO MINERAL; SERVIÇO GEOLÓGICO DO BRASIL - CPRM. Respostas do Serviço Geológico do Brasil aos questionamentos da Braskem. Rio de Janeiro: RJ.

Disponível em: https://rigeo.cprm.gov.br/bitstream/doc/21212/1/respostas_finalizacao_braskem.pdf. Acesso em: 13 jul. 2021.

VASSILEVA, M.; AL-HALBOUNI, D.; MOTAGH, M.; WALTER, T. R.; DAHM, T.; WETZEL, H. A decade-long silent ground subsidence hazard culminating in a metropolitan disaster in Maceió, Brazil. Scientific Reports: nature research. Abr. 2021. DOI: https://doi. org/10.1038/s41598-021-87033-0. Disponível em: https://www.nature.com/articles/s41598-02187033-0.pdf. Acesso em: 13 jul. 2021.

NOVOS equipamentos reforçam monitoramento da área de subsidência. Secretaria Adjunta Especial de Defesa Civil, Maceió. 31 dez 2020. Disponível em: http://www.maceio. al.gov.br/2020/12/novos-equipamentos-reforcam-monitoramento-da-area-de-subsidencia/. Acesso em: 14 jul. 2021.

CASO Pinheiro: a maior tragédia que o Brasil já evitou. Conselho Nacional de Justiça. 29 jan. 2021. Disponível em: https://www.cnj.jus.br/caso-pinheiro-a-maior-tragedia-que-o-brasil-jaevitou/. Acesso em: 12 jul. 2021. 


\section{editoraomnisscientia@gmail.com M} https://editoraomnisscientia.com.br/

@editora_omnis_scientia @ https://www.facebook.com/omnis.scientia.9 f

$$
\text { +55 (87) 9656-3565 @ }
$$




\section{editoraomnisscientia@gmail.com M} https://editoraomnisscientia.com.br/ $\oplus$

@editora_omnis_scientia@ https://www.facebook.com/omnis.scientia.9 f

$$
\text { +55 (87) 9656-3565 @ }
$$

